\title{
The Movement of Orbits and Their Effect on the Encoding of Letters in Partition Theory
}

\author{
Rahmah J. Shareef, Ammar S. Mahmood* \\ University of Mosul, Department of Mathematic, College of Education for Pure Science, Mosul, Iraq \\ Email: *asmahmood65@yahoo.fr
}

How to cite this paper: Shareef, R.J. and Mahmood, A.S. (2019) The Movement of Orbits and Their Effect on the Encoding of Letters in Partition Theory. Open Access Library Journal, 6: e5834.

https://doi.org/10.4236/oalib.1105834

Received: October 8, 2019

Accepted: November 5, 2019

Published: November 8, 2019

Copyright $\odot 2019$ by author(s) and Open Access Library Inc.

This work is licensed under the Creative Commons Attribution International License (CC BY 4.0).

http://creativecommons.org/licenses/by/4.0/

\begin{abstract}
This research aims to study the movement of orbits proposed by Mohammed et al. in 2015 and 2016, and their impact on the encoding of letters adopted by Mahmood and Mahmood in 2019 in order to make the latter more difficult when read in the theory of partition.
\end{abstract}

\section{Subject Areas}

Mathematical Analysis

\section{Keywords}

Partition Theory, Encoding, E-Abacus Diagram

\section{Introduction}

Let $r$ be a non-negative integer. A partition $\mu=\left(\mu_{1}, \mu_{2}, \cdots, \mu_{n}\right)$ of $r$ is a sequence of non-negative integers such that $|\mu|=\sum_{i=1}^{n} \mu_{i}=r$ and for all $i \geq 1$, $\mu_{i} \geq \mu_{i+1}$. Fix $\mu$ is a partition of $r$, choosing an integer $b$ greater than or equal to the number of parts of $\mu$ and defining $\beta_{i}=\mu_{i}+b-i, 1 \leq i \leq b$. The set $\left\{\beta_{1}, \beta_{2}, \cdots, \beta_{b}\right\}$ is said to be the set of $\beta$-numbers for $\mu$, see [1].

Let $e$ be a positive integer number greater than or equal to 2 , we can represent $\beta$-numbers by a diagram called $e$-Abacus diagram.

\begin{tabular}{cccc}
\hline run.1 & run.2 & $\ldots$ & run.e \\
\hline 0 & 1 & $\ldots$ & $\mathrm{e}-1$ \\
$\mathrm{e}$ & $\mathrm{e}+1$ & $\ldots$ & $2 \mathrm{e}-1$ \\
$2 \mathrm{e}$ & $2 \mathrm{e}+1$ & $\ldots$ & $3 \mathrm{e}-1$ \\
$\vdots$ & $\vdots$ & $\vdots$ & $\vdots$ \\
\hline
\end{tabular}

e-Abacusdiagram. 
where every $\beta$ will be represented by a bead $(\bullet)$ which takes its location e-abacus diagram [2].

\section{Orbits}

The logic by which the value of partition is found has led some researchers to choose the concept of orbits for any form of e-abacus diagrams, see [3] and [4].

The basic idea of forming these orbits where $e \geq 2$ then $e$-abacus diagram will appear in an order that is very similar to (matrix mode). Now, if we consider that all that exists within the outer frame of this (matrix) frame is a second orbit and so we will have Table 1 .

Table 1. The relation between the value of e and the number of orbits.

\begin{tabular}{lc}
\hline E & No. of orbits \\
\hline 2 & 1 \\
3 & 2 \\
4 & 2 \\
5 & 3 \\
6 & 3 \\
$\vdots$ & $\vdots$ \\
\hline
\end{tabular}

For example, if $e=3$. See Figure 1 .

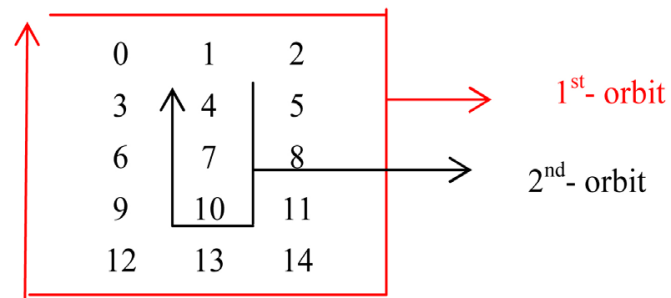

Figure 1. The relation between the value of e and the number of orbits.

And if $e=4$. See Figure 2 .

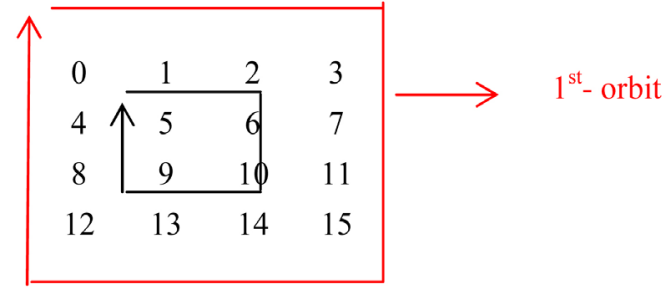

Figure 2. The relation between the value of e and the number of orbits.

This concept will be applied with the coding that has been adopted on the English letters by Mahmood and Mahmood in [5] and [6]. 


\section{Encoding English Letters}

It is well known that there is a lot of research concerning coding or encoding on English letters, they all depend on each letter having a corresponding number from 0 to 25 , hence a particular process begins with special conditions and takes a concept (mod 26), with the exception of Mahmood and Mahmood [5] and [6], they made a change to this concept through the use of e-abacus diagram away from the concept of $(\bmod 26)$, as shown in Table 2.

Table 2. The partition of each English letters.

\begin{tabular}{cccc}
\hline Letters & Partition & Letters & Partition \\
\hline A & $\left(11,8^{2}, 5^{7}, 2,1^{3}\right)$ & B & $\left(11^{3}, 10,8,6^{3}, 5,3,1^{3}\right)$ \\
C & $\left(13^{3}, 12,9,5^{2}, 1^{3}\right)$ & D & $\left(12^{3}, 11,9,8,6,5,3,1^{3}\right)$ \\
E & $\left(12^{4}, 8,6^{3}, 2,1^{4}\right)$ & F & $\left(12,8,6^{3}, 2,1^{4}\right)$ \\
G & $\left(11^{3}, 10,7^{4}, 6,2,1^{3}\right)$ & H & $\left(13,11,10,8,7^{4}, 6,4,3,1\right)$ \\
I & $\left(15^{3}, 12,8,4,1^{3}\right)$ & J & $\left(14,11,10,8,4,1^{3}\right)$ \\
K & $\left(15,13,11,10,7^{2}, 5,4,3,1\right)$ & L & $\left(17^{4}, 13,9,5,1\right)$ \\
M & $\left(12,9^{2}, 8,7^{2}, 6,5^{2}, 4,3,2,1\right)$ & N & $\left(11,9,8^{2}, 7,6^{4}, 5,4^{2}, 3,1\right)$ \\
O & $\left(12^{3}, 11,8^{2}, 5^{2}, 2,1^{3}\right)$ & P & $\left(11^{3}, 8,6^{3}, 5,3,1^{3}\right)$ \\
Q & $\left(11^{4}, 10^{2}, 8^{2}, 5^{2}, 2,1^{3}\right)$ & R & $\left(13,11,10,8,6^{3}, 5,3,1^{3}\right)$ \\
S & $\left(13^{3}, 12,7^{2}, 2,1^{3}\right)$ & T & $\left(14,10,6,2,1^{5}\right)$ \\
U & $\left(14^{2}, 12,10,9,7,6,4,3,1\right)$ & V & $\left(16,13,12,11,8^{2}, 5\right)$ \\
W & $\left(14,13,12,11,10^{2}, 9,8^{2}, 5\right)$ & X & $(13,10,9,8,5,2,1)$ \\
Y & $\left(16,12,9^{3}, 8,5\right)$ & Z & $\left(13^{5}, 10,7,4,1^{4}\right)$ \\
\hline
\end{tabular}

Note that, they are based on the value of $e$ and the number of rows is equal to 5, for example $\mathrm{R}$ and $\mathrm{H}$ write.
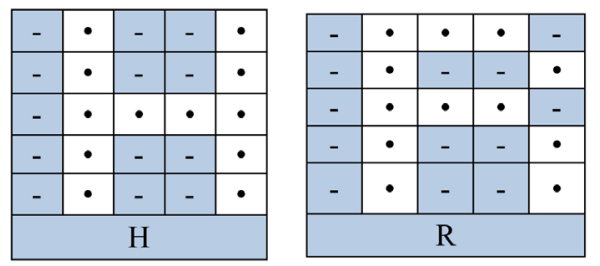

Based on Figure 3, we have three orbits for each letter as shown below.

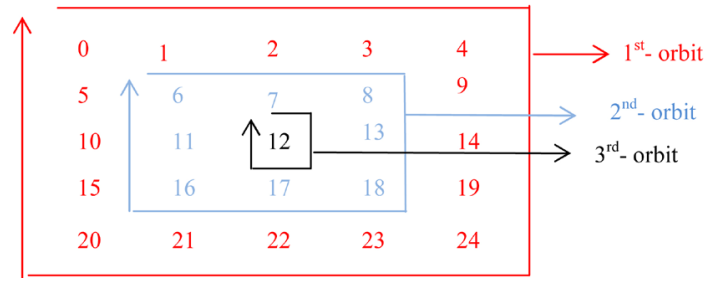

Figure 3. The relation between the value of e and the number of orbits. 


\subsection{Behavior of Each Orbit in $e=5$}

1) 1st-orbit: It is the outer orbit and the largest of all orbits where are 16 sites, we can change the locations according to the clock with one movement, two movements, ... and up to 16 movements until we reach the origin of orbit. Since we always need to have the first location of this orbit empty so we can read the partition; see Mahmood in [7], we cancel this course and keep it as it is to preserve the overall frame of the form of partition.

2) 2nd-orbit: It is the middle orbit and has 8 locations.

3) 3rd-orbit: It is the last orbit that is usually fixed in place because it contains only one location.

If we assume that $w_{t}$ is the motion of the $t$-orbit where $t=1,2,3$, then we have the following.

$\left[w_{1} ; w_{2} ; w_{3}\right]$ such that

$$
w_{1}=w_{3}=0 \text { and } w_{2}=0,1, \cdots, 7 \operatorname{or}\left(w_{2} \bmod 8\right)
$$

\section{2. $w_{2}=1$}

The locations of the 2nd-orbit are

\begin{tabular}{lll}
\hline $\mathrm{a}_{22}$ & $\mathrm{a}_{23}$ & $\mathrm{a}_{24}$ \\
$\mathrm{a}_{32}$ & & $\mathrm{a}_{34}$ \\
$\mathrm{a}_{42}$ & $\mathrm{a}_{43}$ & $\mathrm{a}_{44}$ \\
\hline
\end{tabular}

Its movement will be according to Figure 4 .

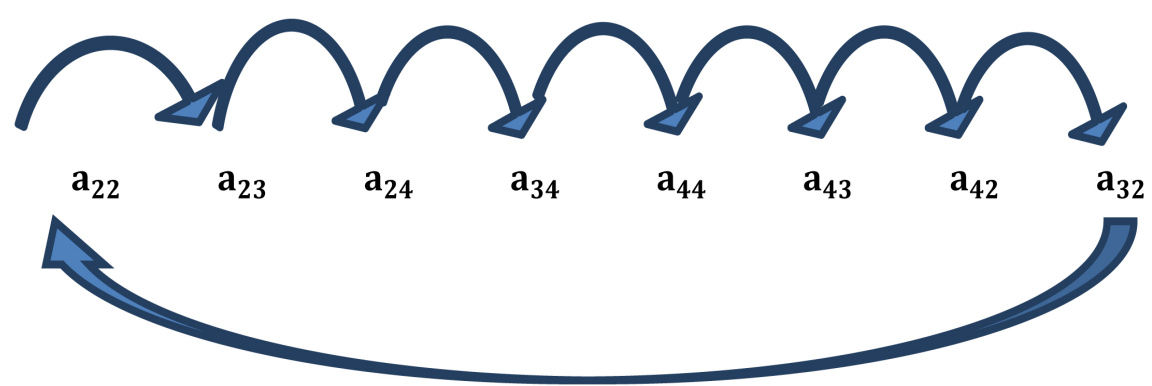

Figure 4. The movement when $\mathrm{w}_{2}=1$.

Thus, we can make the following proposition:

Rule 3.2.1: when choosing a partition for any letter of the English language where $e=5$ and the value of $\beta_{i}$ was equal to the location $a_{\alpha \lambda}$ within $[0 ; 1$; 0] will be

$$
\begin{aligned}
& a_{\alpha \lambda} \rightarrow a_{\alpha(\lambda \mp 1)}, \forall \alpha=2(\text { or } 4) \wedge \lambda=2,3(\text { or } 3,4) \text { respectively } \\
& a_{\alpha \lambda} \rightarrow a_{(\alpha \mp 1) \lambda}, \forall \alpha=2,3(\text { or } 3,4) \wedge \lambda=2(\text { or } 4) \text { respectively }
\end{aligned}
$$

Then, we have Table 3. 
Table 3. The partition of each English letter after application $\mathrm{w}_{2}=1$.

\begin{tabular}{|c|c|c|c|}
\hline Letter & Origin partition & {$[0 ; 1 ; 0]$} & Partition when $w_{2}=1$ \\
\hline A & $\left(11,8^{2}, 5^{7}, 2,1^{3}\right)$ & $\mathrm{A}^{\left[w_{1}, 1, w_{3}\right]}$ & $\left(11,8^{3}, 6^{2}, 5,4^{2}, 2^{2}, 1^{3}\right)$ \\
\hline B & $\left(11^{3}, 10,8,6^{3}, 5,3,1^{3}\right)$ & $\mathrm{B}^{\left[w_{1}, 1, w_{3}\right]}$ & $\left(11^{3}, 10^{2}, 5^{2}, 4,3^{2}, 1^{3}\right)$ \\
\hline $\mathrm{C}$ & $\left(13^{3}, 12,9,5^{2}, 1^{3}\right)$ & $C^{\left[w_{1}, 1, w_{3}\right]}$ & $\left(13^{3}, 12,9,5^{2}, 2,1^{3}\right)$ \\
\hline $\mathrm{D}$ & $\left(12^{3}, 11,9,8,6,5,3,1^{3}\right)$ & $\mathrm{D}^{\left[w_{1}, 1, w_{3}\right]}$ & $\left(12^{3}, 11,7,5,4,3^{2}, 1^{3}\right)$ \\
\hline $\mathrm{E}$ & $\left(12^{4}, 8,6^{3}, 2,1^{4}\right)$ & $\mathrm{E}^{\left[w_{1}, 1, w_{3}\right]}$ & $\left(12^{4}, 10,5^{2}, 2^{2}, 1^{4}\right)$ \\
\hline $\mathrm{F}$ & $\left(12,8,6^{3}, 2,1^{4}\right)$ & $\mathrm{F}^{\left[w_{1}, 1, w_{3}\right]}$ & $\left(12,10,5^{2}, 2^{2}, 1^{4}\right)$ \\
\hline G & $\left(11^{3}, 10,7^{4}, 6,2,1^{3}\right)$ & $\mathrm{G}^{\left[w_{1}, 1, w_{3}\right]}$ & $\left(11^{3}, 10^{2}, 8^{2}, 7,6,2,1^{3}\right)$ \\
\hline $\mathrm{H}$ & $\left(13,11,10,8,7^{4}, 6,4,3,1\right)$ & $\mathrm{H}^{\left[w_{1}, 1, w_{3}\right]}$ & $\left(13,11,10^{2}, 7,6^{2}, 5,4^{2}, 3,1\right)$ \\
\hline I & $\left(15^{3}, 12,8,4,1^{3}\right)$ & $I^{\left[w_{1}, 1, w_{3}\right]}$ & $\left(15^{3}, 11,8,5,1^{3}\right)$ \\
\hline $\mathrm{J}$ & $\left(14,11,10,8,4,1^{3}\right)$ & $J^{\left[w_{1}, 1, w_{3}\right]}$ & $\left(14,10^{2}, 8,5,1^{3}\right)$ \\
\hline $\mathrm{K}$ & $\left(15,13,11,10,7^{2}, 5,4,3,1\right)$ & $\mathrm{K}^{\left[w_{1}, 1, w_{3}\right]}$ & $\left(15,13,10,7^{3}, 4^{2}, 3,1\right)$ \\
\hline $\mathrm{L}$ & $\left(17^{4}, 13,9,5,1\right)$ & $\mathrm{L}^{\left[w_{1}, 1, w_{3}\right]}$ & $\left(17^{4}, 8,5^{2}, 1\right)$ \\
\hline M & $\left(12,9^{2}, 8,7^{2}, 6,5^{2}, 4,3,2,1\right)$ & $\mathbf{M}^{\left[w_{1}, w_{3}\right]}$ & $\left(12,9^{2}, 7^{3}, 6,5^{3}, 3,2,1\right)$ \\
\hline $\mathrm{N}$ & $\left(11,9,8^{2}, 7,6^{4}, 5,4^{2}, 3,1\right)$ & $\mathrm{N}^{\left[w_{1}, 1, w_{3}\right]}$ & $\left(11,9,8^{3}, 6,5^{2}, 4^{4}, 3,1\right)$ \\
\hline $\mathrm{O}$ & $\left(12^{3}, 11,8^{2}, 5^{2}, 2,1^{3}\right)$ & $\mathrm{O}^{\left[w_{1}, 1, w_{3}\right]}$ & $\left(12^{3}, 11,8^{2}, 5^{2}, 2,1^{3}\right)$ \\
\hline $\mathrm{P}$ & $\left(11^{3}, 8,6^{3}, 5,3,1^{3}\right)$ & $\mathrm{P}^{\left[w_{1}, 1, w_{3}\right]}$ & $\left(11^{3}, 10,5^{2}, 4,3^{2}, 1^{3}\right)$ \\
\hline Q & $\left(11^{4}, 10^{2}, 8^{2}, 5^{2}, 2,1^{3}\right)$ & $\mathrm{Q}^{\left[w_{1}, 1, w_{3}\right]}$ & $\left(11^{4}, 10,9,8^{2}, 5^{2}, 2,1^{3}\right)$ \\
\hline $\mathrm{R}$ & $\left(13,11,10,8,6^{3}, 5,3,1^{3}\right)$ & $\mathrm{R}^{\left[w_{1}, 1, w_{3}\right]}$ & $\left(13,11,10^{2}, 5^{2}, 4,3^{2}, 1^{3}\right)$ \\
\hline S & $\left(13^{3}, 12,7^{2}, 2,1^{3}\right)$ & $\mathrm{S}^{\left[w_{1}, 1, w_{3}\right]}$ & $\left(13^{3}, 11,7,2^{2}, 1^{3}\right)$ \\
\hline $\mathrm{T}$ & $\left(14,10,6,2,1^{5}\right)$ & $\mathrm{T}^{\left[w_{1}, 1, w_{3}\right]}$ & $\left(14,9,6,3,1^{5}\right)$ \\
\hline $\mathrm{U}$ & $\left(14^{2}, 12,10,9,7,6,4,3,1\right)$ & $\mathrm{U}^{\left[w_{1}, 1, w_{3}\right]}$ & $\left(14^{2}, 12,8,6,5,4^{2}, 3,1\right)$ \\
\hline $\mathrm{V}$ & $\left(16,13,12,11,8^{2}, 5\right)$ & $\mathrm{V}^{\left[w_{1}, 1, w_{3}\right]}$ & $\left(16,12,10,8^{3}, 5\right)$ \\
\hline $\mathrm{W}$ & $\left(14,13,12,11,10^{2}, 9,8^{2}, 5\right)$ & $\mathrm{W}^{\left[\mathrm{w}_{1}, 1, w_{3}\right]}$ & $\left(14,13,12,10^{3}, 9,8^{2}, 5\right)$ \\
\hline $\mathrm{X}$ & $(13,10,9,8,5,2,1)$ & $X^{\left[w_{1}, 1, w_{3}\right]}$ & $\left(13^{2}, 11,5,4,2,1\right)$ \\
\hline $\mathrm{Y}$ & $\left(16,12,9^{3}, 8,5\right)$ & $\mathrm{Y}^{\left[w_{1}, 1, w_{3}\right]}$ & $\left(16,13,12,9,7,5^{2}\right)$ \\
\hline Z & $\left(13^{5}, 10,7,4,1^{4}\right)$ & $\mathrm{Z}^{\left[w_{1}, 1, w_{3}\right]}$ & $\left(13^{5}, 7^{3}, 1^{4}\right)$ \\
\hline
\end{tabular}

\section{3. $2 \leq w_{2} \leq 7$}

When $2 \leq w_{2} \leq 7$ and applied to Table 2 separately, we produce the following rule: Rule 3.3.1: when choosing a partition for any letter of English language where $e=5$ and the value of $\beta_{i}$ was equal to the number of location $a_{\alpha \lambda}$ within [0; $1 ; 0]$ will be:

1) If $w_{2}=2$, then we have:

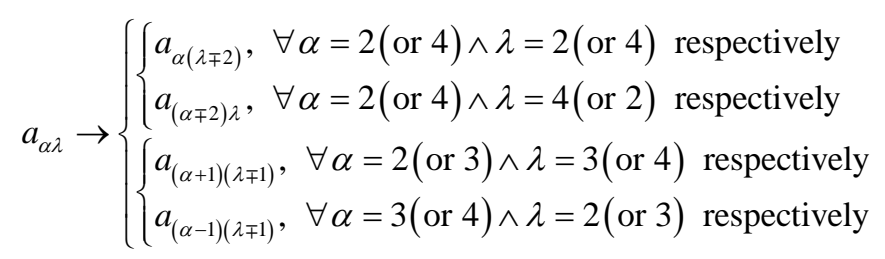


2) If $w_{2}=3$, then

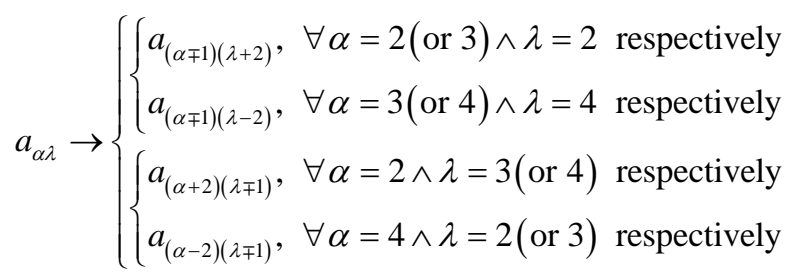

3) If $w_{2}=4$, then

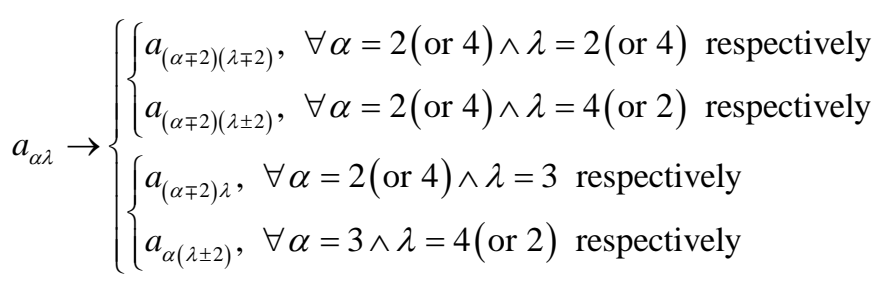

4) If $w_{2}=5$, then

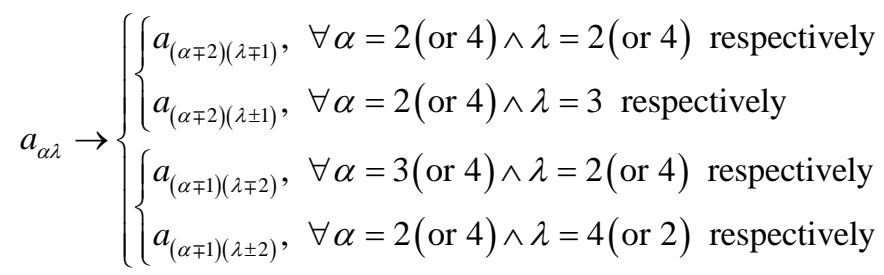

5) If $w_{2}=6$, then

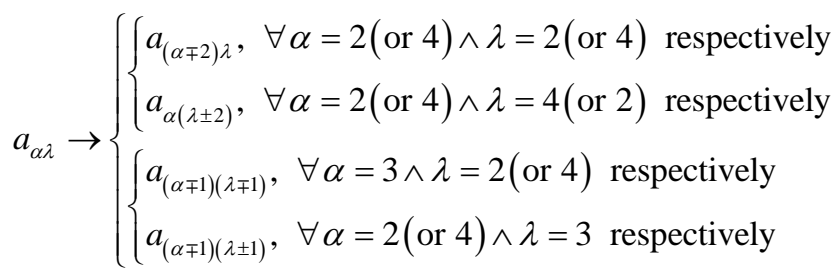

6) If $w_{2}=7$, then

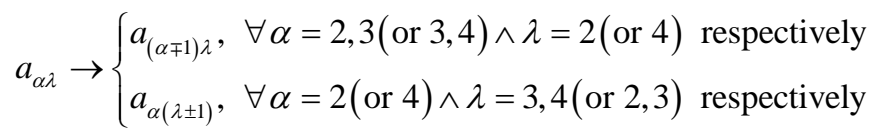

For example (See Table 4).

Table 4. The partition of $\mathrm{R}$ and $\mathrm{N}$ after application $2 \leq \mathrm{w}_{2} \leq 7$.

\begin{tabular}{cccc}
\hline Letter & Partition & Letter & Partition \\
\hline $\mathrm{R}$ & $\left(13,11,10,8,6^{3}, 5,3,1^{3}\right)$ & $\mathrm{N}$ & $\left(11,9,8^{2}, 7,6^{4}, 5,4^{2}, 3,1\right)$ \\
$\mathrm{R}^{\left[w_{1}, 2, w_{3}\right]}$ & $\left(13,11,10,9,5,3^{4}, 1^{3}\right)$ & $\mathrm{N}^{\left[w_{1}, 2, w_{3}\right]}$ & $\left(11,9,8,7^{2}, 6^{3}, 4^{4}, 3,1\right)$ \\
$\mathrm{R}^{\left[w_{1}, 3, w_{3}\right]}$ & $\left(13,11,10,8,6^{2}, 4^{3}, 1^{3}\right)$ & $\mathrm{N}^{\left[w_{1}, w_{3}\right]}$ & $\left(11,9,8^{2}, 7,6^{4}, 5^{3}, 3,1\right)$ \\
$\mathrm{R}^{\left[w_{1}, 4, w_{3}\right]}$ & $\left(13,11,10^{2}, 6^{3}, 5^{2}, 1^{3}\right)$ & $\mathrm{N}^{\left[w_{1}, 4, w_{3}\right]}$ & $\left(11,9,8^{3}, 6^{4}, 5^{2}, 4,3,1\right)$ \\
$\mathrm{R}^{\left[w_{1}, 5, w_{3}\right]}$ & $\left(13,11,10^{3}, 7^{2}, 5,3,1^{3}\right)$ & $\mathrm{N}^{\left[w_{1}, 5, w_{3}\right]}$ & $\left(11,9,8^{4}, 7^{3}, 5,4^{2}, 3,1\right)$ \\
$\mathrm{R}^{\left[w_{1}, 6, w_{3}\right]}$ & $\left(13,11,10^{4}, 7,5,4,1^{3}\right)$ & $\mathrm{N}^{\left[w_{1}, 6, w_{3}\right]}$ & $\left(11,9,8^{4}, 7,6^{2}, 5^{3}, 3,1\right)$ \\
$\mathrm{R}^{\left[w_{1}, 7, w_{3}\right]}$ & $\left(13,11,10,9^{2}, 6^{2}, 5^{2}, 1^{3}\right)$ & $\mathrm{N}^{\left[w_{1}, 7, w_{3}\right]}$ & $\left(11,9,8,7^{2}, 6^{4}, 5^{2}, 4,3,1\right)$ \\
\hline
\end{tabular}




\section{Conflicts of Interest}

The authors declare no conflicts of interest regarding the publication of this paper.

\section{References}

[1] Mathas (1999) Iwahori-Hecke Algebras and Schur Algebras of the Symmetric Group. Univ. Lecture Series, Vol. 15.

http://citeseerx.ist.psu.edu/viewdoc/download?doi=10.1.1.49.9949\&rep=rep1\&type $=$ pdf https://doi.org/10.1090/ulect/015/02

[2] James, G. (1978) Some Combinatorial Results Involving Young Diagrams. Mathematical Proceedings of the Cambridge Philosophical Society, 83, 1-10.

https://www.cambridge.org/core/journals/mathematical-proceedings-of-the-cambri dge-philosophical-society/article/some-combinatorial-results-involving-young-diag rams/2F5541FEF300385926F88064F5E98F04

https://doi.org/10.1017/S0305004100054220

[3] Mahommed, E.F., Ahmad, N., Ibrahim, H. and Mahmood, A.S. (2015) Embedding Chain Movement in James Diagram for Partitioning Beta Number. AIP Conference Proceedings, 1691, 040019. https://aip.scitation.org/doi/10.1063/1.4937069 https://doi.org/10.1063/1.4937069

[4] Mahommed, E.F., Ahmad, N., Ibrahim, H. and Mahmood, A.S. (2016) Nested Chain Movement of Length 1 of Beta Number in James Abacus Diagram. Global Journal of Pure and Applied Mathematics, 12, 2953-2969. https://www.ripublication.com/gjpam16/gjpamv12n4 17.pdf

[5] Mahmood, A.B. and Mahmood, A.S. (2019) Secret-Word by e-Abacus Diagram I. Iraqi Journal of Science, 60, 638-646.

https://www.researchgate.net/publication/332058738 Secret-word by e-abacus dia gram I

[6] Mahmood, A.B. and Mahmood, A.S. (2019) Secret-Text by e-Abacus Diagram II. Iraqi Journal of Science, 60, 840-846.

https://www.researchgate.net/publication/332786557 Secret-text by e-abacus diag ram II

[7] Mahmood, A.S. (2011) On the Intersection of Young's Diagrams Core. Journal of Education and Science, 24, 149-157.

https://www.iasj.net/iasj?func=fulltext\&aId $=58795$

https://doi.org/10.33899/edusj.1999.58795 